\title{
Innovative Design of Solar-Powered Desalination (SPD) System using Vacuum-Multi Effect Membrane Distillation (V-MEMD) Process
}

\author{
Achmad Chafidz ${ }^{1, *}$, Faisal RM ${ }^{1}$, Esa D. Kerme ${ }^{2}$, Irfan Wazeer $^{3}$, Saeed M. AlZahrani ${ }^{3}$ \\ ${ }^{1}$ Department of Chemical Engineering, Universitas Islam Indonesia, Yogyakarta 55584, Indonesia \\ ${ }^{2}$ Mechanical Engineering Department, King Saud University, Riyadh 11421, Saudi Arabia \\ ${ }^{3}$ Chemical Engineering Department, King Saud University, Riyadh 11421, Saudi Arabia
}

\begin{abstract}
This research focused on the development of an innovative design of solar-powered desalination (SPD) system which was expected to solve the water and energy problem simultaneously. We have developed a portable and hybrid solar-powered desalination (SPD) system for producing potable water from saline water. It is a self-contained and integrated system which combines solar-thermal collector and solar-photovoltaic for its operation, and thus the system can operate to produce water by only using solar energy. Therefore, the system is highly suitable to be implemented in remote arid and coastal areas without infrastructures or connection to the grid (water and power), but blessed with abundant solar irradiation, like in Saudi Arabia. A Memsys Vacuum Multi-Effect Membrane Distillation (V-MEMD) unit was used as the core of the SPD system. A heat pump was also integrated into the SPD system for energy recovery and to improve the performance of the system. The system could be considered as sustainable and "green" desalination technology, which will be very useful for the Kingdom of Saudi Arabia. To study the performance of the system, small-scale tests have been carried out at the Engineering College - King Saud University, Saudi Arabia. Based on the experimental results, the system has run successfully by only utilizing solar energy.
\end{abstract}

\section{Introduction}

Fresh water is one of the most important human needs besides food and energy. Without three of them (i.e. water, food, energy) human cannot live normally. Additionally, three of them also interdependent with each other. Energy crisis will lead to water crisis. This is because to produce water requires a lot of energy, for example: for seawater desalination, water treatment, water distribution (by pump), etc. In reverse, to produce energy also require a large amount of water, for example: cooling, power plant, oil production, etc. On the other hand, water crisis will inevitably lead to food crisis. The food crisis cannot be denied will also lead to a decline in the standard of human life, which will affect the performance of their work, e.g. in the energy industrial sector. The relationship/interdependence between these three is what is called by "The Nexus of Water, Energy, and Food".

It is already known that most of the Middle East and North Africa (MENA) countries, such as Kingdom of Saudi Arabia (KSA) has limited amount of natural fresh water resources. In contrary, Indonesia, an archipelago country has abundant natural fresh water resources, such as: lake, river, underground water, rain, etc. The most promising method and maybe the only option for a country such as Saudi Arabia to be able to meet the fresh water needs for its citizens is by seawater desalination. It is because seawater resources is very abundant, about $96 \%$ of earth's total water distribution is seawater. To meet the water demand, Saudi Arabia has constructed numbers of large scale desalination plants, which accounted for about $24 \%$ of world total capacity. The number of the desalination plant will definitely increase with increasing its population. According to figures from Electricity \& Cogeneration Regulatory Authority (ECRA) of Saudi Arabia, most of desalination plants in Saudi Arabia are using conventional technologies, such as Multi-Stage Flash process (MSF), Reverse Osmosis (RO), and Multi-Effect Distillation (MED). These conventional processes required significant amount of energy and most of them are driven by fossil fuel. As one of the top oil producers in the world, maybe Saudi Arabia does not have a problem with the water supply through desalination plant driven by fossil fuel. However, in the future, when the oil resources are depleted then the energy crisis will inevitably fall upon this kingdom, which will then lead to water crisis. One the most promising alternative to solve the water and energy problem simultaneously is by utilizing renewable energy sources to power seawater desalination process. One of the most potential renewable energy in Saudi

\footnotetext{
* Corresponding author: achmad.chafidz@uii.ac.id
} 
Arabia is solar energy. Therefore, utilizing solar energy to drive seawater desalination process may become the best alternative for Saudi Arabia to meet the domestic water demand as well as reducing the oil consumption.

Membrane distillation (MD) is one among desalination technologies that is compatible to be coupled with the solar energy. The MD process can be described as combined process between membrane and thermal distillation proceses. It can also be called a "trans-membrane evaporation" process [1-3]. The basic working principle of MD process is very simple. Heat is applied in the MD process to generate vapor from the saline water. The MD uses microporous hydrophobic membrane for its separation process. Due to its hydrophobic nature of the membrane, only vapor can pass through the membrane, and thus separating the (pure) water from the saline water. There are several advantages of MD process, such as: the process is very simple, it can be used for any range of saline water, less fouling tendency, the heat used can be low grade heat, it can produce water with high quality, it can be run with small temperature difference $[1,2,4,5]$. Based on the mechanism, the MD process can be classified into several different configurations. Figure 1 shows the schematic illustration of three different configurations of MD process. In Vacuum membrane distillation (VMD), a vacuum is applied to the permeate side and the vapor condensation occurs outside the membrane. This vacuum will help the vaporization process to be started at low temperature. In Direct contact membrane distillation (DCMD), inside the permeate side is a condensing liquid, which in direct contact with the membrane. Whereas, in AGMD, the condensing (cold) surface is separated from the membrane by an air gap [3].
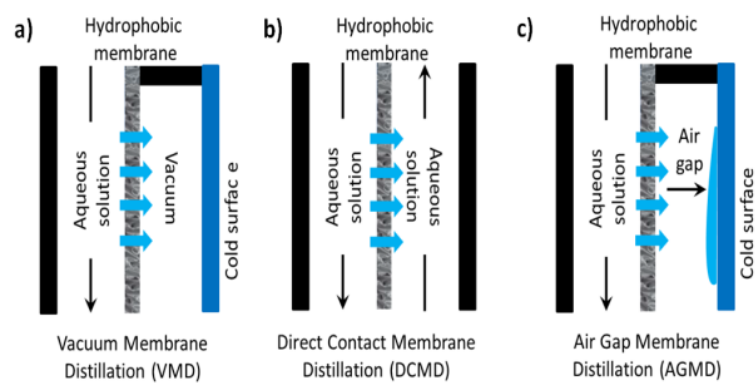

Fig. 1. Illustration of three different configurations of membrane distillation process: a) VMD; b) DCMD; and AGMD. Adopted from the literature [1].

\section{The SPD system configuration}

The solar-powered desalination (SPD) system is a selfcontained and integrated system which combines solarthermal collector and solar-photovoltaic for its operation. Thus, the system can operate to produce water by only using solar energy. For producing the water, a vacuummulti effect membrane distillation (V-MEMD) unit (Memsys, Germany) was used. Figure 2 (Adopted from the literature [1]) shows the schematic diagram of the SPD system. The SPD system consists of three main sub-systems, which are: 1) Solar-photovoltaic (PV) system, to harness solar energy and converts into electricity for powering the equipment; 2) Solar-thermal collector system, to harness solar energy and converts into thermal energy to supply the heat required by the Memsys unit via a heat exchanger; and 3) Memsys VMEMD system for producing the water by utilizing the low grade heat generated by the thermal collector. As seen in Fig. 2, the SPD system was also equipped with a

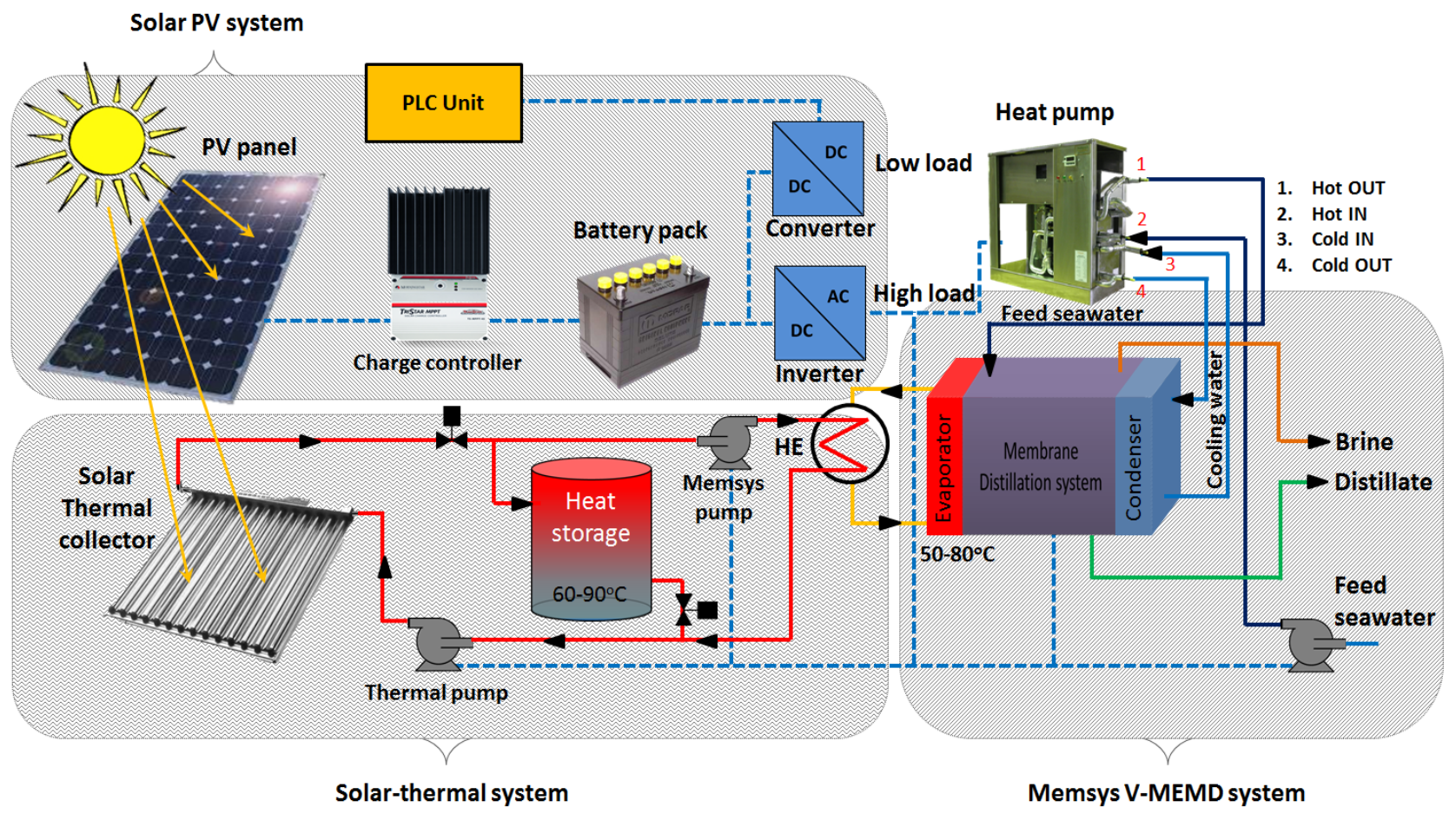

Fig. 2. Schematic diagram of the SPD system. 
heat pump, which was used for energy recovery by cools the cooling water (in the condenser loop) as well as preheats the feed water (e.g. seawater, brackish water) during operation of the SPD system. The more details of the SPD system could be found in our previous work [1, 6].

Additionally, the system was also designed to be able to operate in arid remote areas. This advantage is coming from the design of the system which utilizes a typical modified container $(6.0 \mathrm{~m} \mathrm{x} 2.4 \mathrm{~m} \mathrm{x} 2.6 \mathrm{~m})$ to house all the equipments, thus it is portable and can be deployed at anytime. The seawater tank is also housed in one side of the container. This seawater is used for feed as well as for cooling for the Memsys V-MEMD unit. The seawater was prefiltered using an ultrafiltration cartridge when filling the seawater tank. Figure 3 shows the photograph of the SPD system. Currently the SPD system was placed on the rooftop of College Engineering building, at King Saud University, Riyadh, Kingdom of Saudi Arabia.

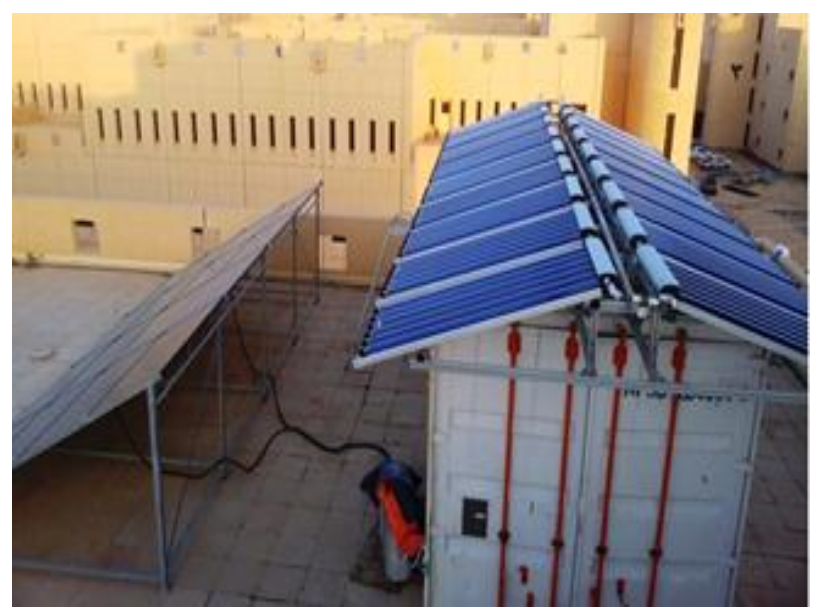

Fig. 3. Photographs of the SPD system

The Memsys Vacuum Multi-Effect Membrane Distillation (V-MEMD) unit is the core of the SPD system. The main purpose of this unit/sub-system is to produce a clean water by utilizing the low grade heat generated by the solar-thermal collector. The Memsys unit is desalination process that combines both the thermal process (i.e. multi-effect distillation) and membrane separation process. In general, the working principle of the Memsys unit is based on the Vacuum Multi-Effect Membrane Distillation (V-MEMD) process. According to the literature [7], the Memsys V-MEMD process has advantages due to some reasons, such as: internal heat recovery, high distillate flux, minimum heat loss, low specific heat transfer surface, low mechanical stress due to less pressure difference on membrane from feed stream and negative pressure, modularity. The last point is important to compete with the many advantages of Reverse Osmosis (RO). In which, the Memsys VMEMD unit, due to its modularity, it has wide range of applications, from small to large scale [7]. Figure 4a shows the photograph of the complete Memsys unit. The unit consists of three major parts: a steam raiser, multiple effect (evaporation-condensation) stages, and a condenser. Please see also Fig. 5b, which shows the configuration of the Memsys unit.
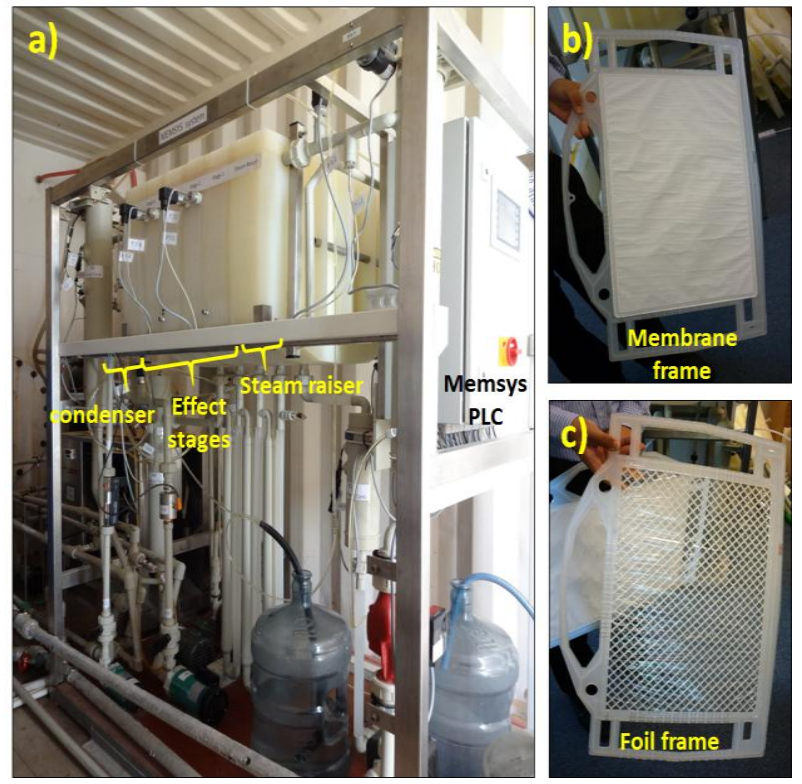

Fig. 4. Photographs of the Memsys unit: a) The complete modul of the Memsys unit; b) The Memsys membrane frame; and c) The Memsys foil frame.

The purpose of the steam raiser is to create steam (from fresh water) which will acts as driving thermal energy source for the operation of Memsys process. To produce steam, the steam rasier utilizes heat from the thermal tank that is transferred through a plate heat exchanger. Due to the vacuum pressure $(<200 \mathrm{mbar})$, the evaporation process could happen with low temperature. In general, the Memsys process can run with temperature in the range of $50-80{ }^{\circ} \mathrm{C}$. Whereas, the evaporation-condensation stages, or can be called the effect stages is where the distillate is produced. The thermal energy from the steam raiser evaporates the seawater and the water vapor generated get separated by the microporous hydrophobic membrane, then condensed by the polypropylene (PP) foil, and finally collected at the bottom of effect stages. There is a multiple recycle of thermal energy, and thus it can reduces the amount of required energy and increases thermal efficiency of the system. In this work, the Memsys unit consists of four effect stages. Each stage consists of four membrane frames and PP foil frames (see Fig. $4 \mathrm{~b}$ and $4 \mathrm{c}$ ), with the total effective membrane area of $5.12 \mathrm{~m}^{2}$. Apparently, this membrane area is smaller than other SPD systems [1]. Figure 5a shows the schematic illustration of how the feed and vapor flows inside one effect stage of the Memsys V-MEMD unit. The last part is a condenser, which its purpose is to condense the water vapor being produced at the last effect stages. A fresh water with temperature of 20-40 ${ }^{\circ} \mathrm{C}$ is used as the coolant. Additionally, the temperature difference between the steam raiser and the condenser is the driving force for the Memsys process. Therefore, the greater the temperature difference, the greater the amount of distillate could be produced by the Memsys 
unit. The more details of the Memsys unit can be found in our previous work [1].

a)

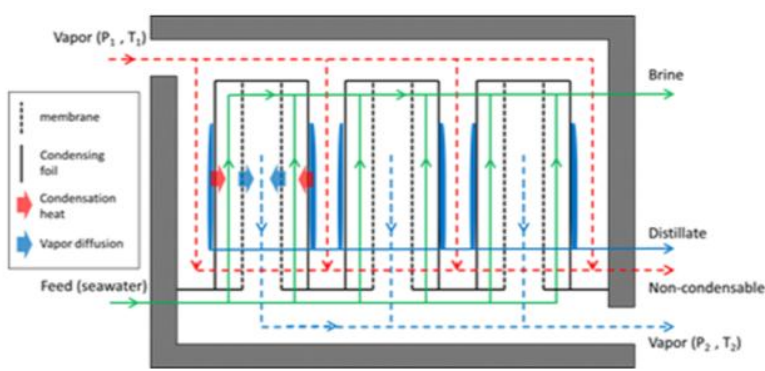

b)

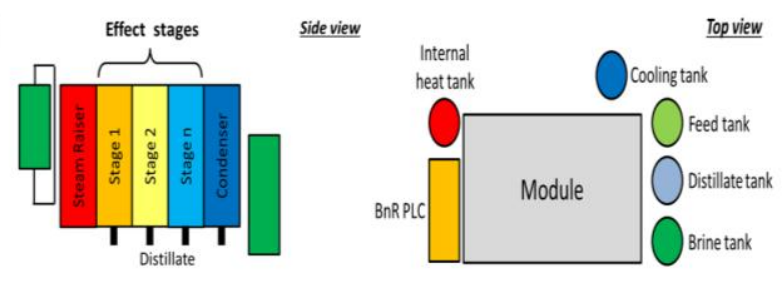

Fig. 5. a) Schematic illustration of how the Memsys V-MEMD process works (inside one effect stage) - Adopted from the literature [7], and b) The schematic configuration of the Memsys unit (side view and top view).

\section{One-day test of the SPD system}

The SPD system was place on the roof-top of college engineering building, King Saud University, Riyadh, Saudi Arabia. It is worth to note that the system was a prototype of solar powered desalination system with a small capacity of water production. Small-scale tests of the system were conducted to observe the performance of the SPD system. Figure 6 shows a one-day operation of the SPD system. The test was conducted on $21 \mathrm{Feb}$ 2013 (spring season). The system was run from 9:00 AM until 4:00 PM (about 7 hours). The system was run in manual mode. The solar irradiation level was monitored all the time using a Rotating Shadowband Radiometer (Irradiance Inc., USA). As seen in Fig. 6, the range of global irradiation level on that day was from 308.5 to $870 \mathrm{~W} / \mathrm{m}^{2}$. The peak level of $870 \mathrm{~W} / \mathrm{m}^{2}$ was occurred at 12:22 AM. The Memsys unit started to produce the water at 9:26 AM. The first half an hour was used to stabilize the SPD system, especially the thermal energy required by the Memsys unit. The amount of time needed to stabilize the system depends on the thermal tank temperature. The higher the initial temperature of the thermal tank, the less amount of time needed for the Memsys unit to start producing the water. In the current test, the initial temperature of thermal tank was already at $72{ }^{\circ} \mathrm{C}$ (see Fig. 6). This temperature was high enough to provide a driving force for the Memsys unit to produce water. During the test, the thermal tank temperature did not change siginificantly. The temperature was only in the range of $72-76.3{ }^{\circ} \mathrm{C}$.

The feed used in this test was a brackish water with conductivity of $2490 \mu \mathrm{S} / \mathrm{cm}$. This conductivity was offline measured using a Multi-Parameter Analyzer (DZS-708), Cheetah Scientific Ltd, Malaysia. The feed flowrate was kept constant at $69 \mathrm{~L} / \mathrm{h}$ during the test. Based on the previous tests performance of the system (see our previous work [6]), this feed flow rate was found to be the optimum rate to produce maximum distillate. Additionally, as seen in Fig. 6, the distillate production rate was in the range of $11.75-20.29 \mathrm{~L} / \mathrm{h}$, with average distillate production rate of $15.91 \mathrm{~L} / \mathrm{h}$. As expected the maximum distillate production rate was occurred at noon, around 12:00 AM, where the global irradiation level also reached its peak. Whereas, the total distillate output gained on that day was approximately 102.9 L of fresh water with approximate conductivity of $4.7 \mu \mathrm{S} / \mathrm{cm}$. This water is clean water and safely to drink. This total volume of distillate output can be considered lower than the other systems (see in the literature ). This is due to small capacity of the Memsys unit, which only has a total membrane area of $5.12 \mathrm{~m}^{2}$. Nevertheless, this is not a problem, since the Memsys unit capacity can be easily increased due to modularity of the Memsys module.

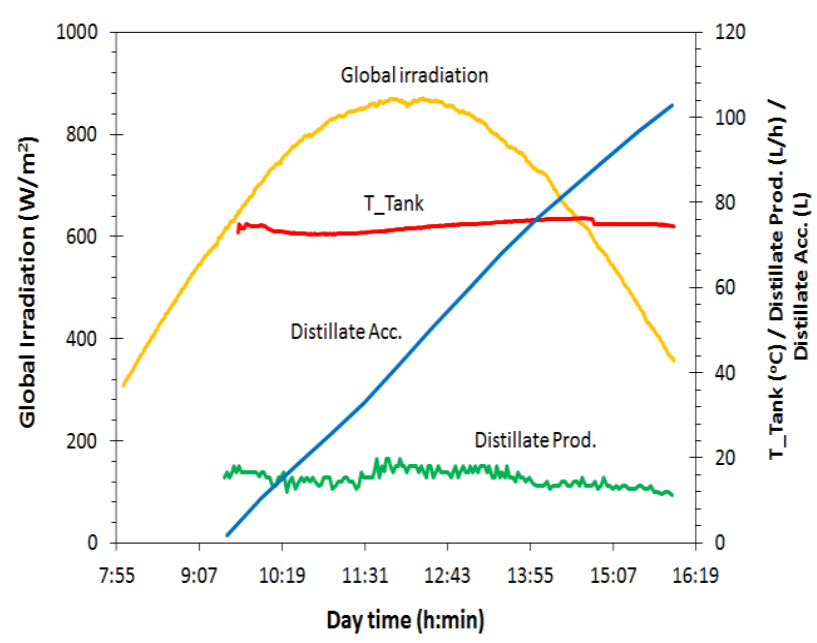

Fig. 6. A one-day performance test of the SPD system.

Additionally, there is one parameter that can be used to compare the performance of the SPD system with the others, proportionally. The parameter is called disitillate flux $(J)$ with the unit of $\mathrm{L} / \mathrm{m}^{2} . \mathrm{h}$, which can be defined by Eq. (1) as follows:

$$
I=\frac{V}{S_{t} t}
$$

where $V$ is the volume of the distillate produced (L), $S$ is the total effective membrane area $\left(\mathrm{m}^{2}\right)$, and $t$ is the running time of the Memsys unit. The total effective membrane area of the Memsys module was approximately $5.12 \mathrm{~m}^{2}$. Figure 7 shows the distillate flux profile of the Memsys unit on the same day of operation (i.e. 21 Feb 2013). As seen in the figure, the profile of distillate flux was following the trend of global irradiation level, which reached its peak at the noon time. The distillate flux was found in the range of $2.29-$ $3.96 \mathrm{~L} / \mathrm{m}^{2}$.h, with the approximate average value of 3.12 $\mathrm{L} / \mathrm{m}^{2}$.h. This distillate flux rate was comparable or even higher than some of other SPD systems [1]. 


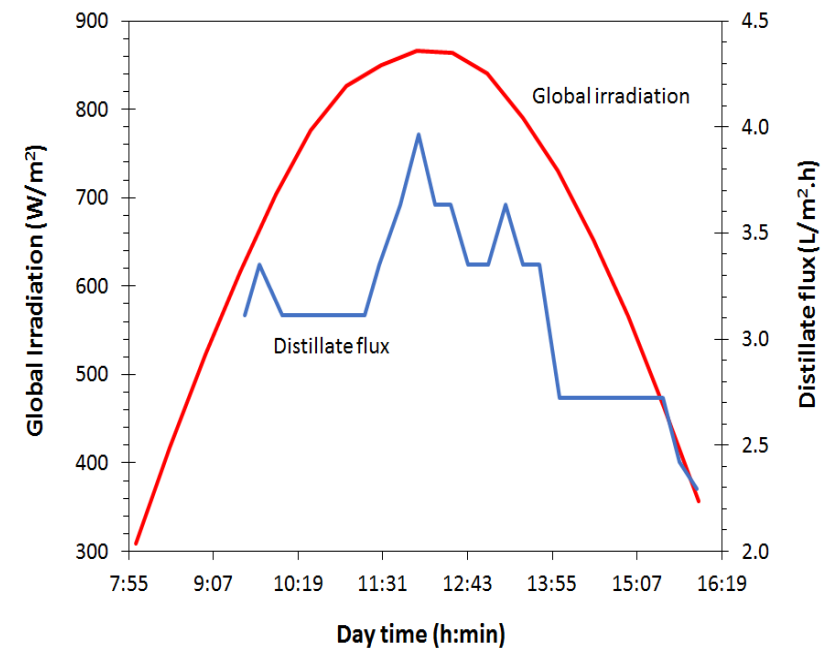

Fig. 7. Hourly distillate flux of the SPD system during one day performance test.

\section{Conclusion}

An innovative solar-powered desalination (SPD) system which is a stand-alone, integrated, and portable has been designed and successfully developed. It is a hybrid system which combines solar-thermal collector and solar-photovoltaic for its operation. In this work, a oneday operation test of the SPD system has been conducted on 21 Feb 2013 (spring season in Saudi Arabia). The test results showed that the SPD system has run successfully by only utilizing solar energy. The system was run from 9:00 AM until 4:00 PM (about 7 hours). The range of global irradiation level on that day was from 308.5 to $870 \mathrm{~W} / \mathrm{m}^{2}$ with the peak level occurred at 12:22 AM. The thermal tank temperature was in the range of $72-$ $76.3{ }^{\circ} \mathrm{C}$. The feed (saline water) was a brackish water with conductivity of $2490 \mu \mathrm{S} / \mathrm{cm}$. The feed flowrate was kept constant at $69 \mathrm{~L} / \mathrm{h}$. The distillate production rate was in the range of $11.75-20.29 \mathrm{~L} / \mathrm{h}$, with average distillate production rate of $15.91 \mathrm{~L} / \mathrm{h}$. The total distillate output gained on that day was approximately $102.9 \mathrm{~L}$ with approximate conductivity of $4.7 \mu \mathrm{S} / \mathrm{cm}$. The distillate flux was found in the range of $2.29-3.96$ $\mathrm{L} / \mathrm{m}^{2} . \mathrm{h}$, with the approximate average value of 3.12 $\mathrm{L} / \mathrm{m}^{2}$.h. This distillate flux rate was comparable or even higher than some of other SPD systems.

This solar-powered desalination (SPD) system has been developed by a joint collaboration project between King Saud University (KSU), Kingdom of Saudi Arabia and Nanyang Technological University (NTU), Singapore. The authors would like to thank all the contributors for this project: Choo F. H., Tan F.L., Darryl, M. Prabu, Naeem.

\section{References}

[1] A. Chafidz, E. D. Kerme, I. Wazeer, Y. Khalid, A. Ajbar, S.M. Al-Zahrani, Journal of Cleaner Production, 133 (2016) 631-647.
[2] M. Safavi, T. Mohammadi, Chem. Eng. J. (Lausanne), 149 (2009) 191-195.

[3] J.-P. Mericq, S. Laborie, C. Cabassud, Chem. Eng. J. (Lausanne), 166 (2011) 596-606.

[4] H. Chang, C.-L. Chang, C.-Y. Hung, T.-W. Cheng, C.-D. Ho, International Journal of Environmental Research and Public Health, 11 (2014) 1206412087.

[5] A. Hausmann, P. Sanciolo, T. Vasiljevic, M. Weeks, M. Duke, Chem. Eng. J. (Lausanne), 211212 (2012) 378-387.

[6] A. Chafidz, S. Al-Zahrani, M.N. Al-Otaibi, C.F. Hoong, T.F. Lai, M. Prabu, Desalination, 345 (2014) 36-49.

[7] W. Heinzl, S. Büttner, G. Lange, Desalination and Water Treatment, 42 (2012) 177-180. 